\title{
ADOMAIN DECOMPOSITION METHOD FOR SOLVING SYSTEMS OF MULTI-DIMENSIONAL LINEAR FREDHOLM INTEGRAL EQUATIONS OF THE SECOND KIND
}

\author{
Ahlam Jameel Khaleel* and Hanan Mahmood Hasoon ${ }^{* *}$ \\ "College of Science, Al-Nahrian University. \\ *** College of Education, Ibn Al-Haithm, University of Baghdad.
}

\begin{abstract}
The aim of this work is to use Adomian decomposition method to solve Systems of multidimensional linear Fredholm integral equations of the second kind.
\end{abstract}

\section{1-Introduction}

Adomain decomposition method was first introduced by Adomain G. in 1980. This method is used to solve differential equations, [1], [2]. The convergence of Adomian decomposition method applied to the onedimensional integral equations is discussed in [5]. Moreover this method is used to solve systems of the one-dimensional Volterra integral equations of first kind, [4], systems of linear equations and systems of the onedimensional Volterra integral equations of second kind, [3], systems of the onedimensional Fredholm integral equations of the second kind, [7] and systems of fractional differential equations, [6]. Here we use this method to solve systems of the multidimensional linear Fredholm integral equations of the second kind:

$u_{i}\left(x_{1}, x_{2}, \ldots, x_{n}\right)=f_{i}\left(x_{1}, x_{2}, \ldots, x_{n}\right)+$

$\int_{a_{1}}^{b_{1}} \int_{a 2}^{b_{2}} \ldots \int_{a n}^{b n} \sum_{j=1}^{n} k_{i, j}\left(x_{1}, x_{2}, \ldots, x_{n}, y_{1}, y_{2}, \ldots, y_{n}\right)$.

$u_{j}\left(y_{1}, y_{2}, \ldots, y_{n}\right) d y_{n} d y_{n-1} \ldots d y_{1}, i=1,2, \ldots, n$

Where $\mathrm{f}_{\mathrm{i}}$ is a known function of $x_{1}, x_{2}, \ldots, x_{n}, k_{i, j}$ is a known function of $x_{1}, x_{2}, \ldots, x_{n}, y_{1}, y_{2}, \ldots, y_{n},\left\{a_{i}\right\}_{i=1}^{n},\left\{b_{i}\right\}_{i=1}^{n} \quad$ are known constants such that $a_{i} \leq x_{i} \leq b_{i}$, $\mathrm{i}=1,2, \ldots, \mathrm{n}$ and $u_{1}, u_{2}, \ldots, u_{n}$ are the unknown functions that must be determined.

\section{2- Adomain Decomposition Method Applied} to $\operatorname{System(1)}$

Consider the system of the multidimensional linear Fredholm integral equations of the second kind given by equation (1).
We rewrite this equation as a canonical form of Adomain's equation by letting

$$
\begin{aligned}
& N_{i}\left(x_{1}, x_{2}, \ldots, x_{n}\right)=\int_{a_{1} a 2}^{b_{1} b_{2}} \ldots \int_{a n j}^{b n} \sum_{j=1}^{n} k_{i, j}\left(x_{1}, x_{2}, \ldots, x_{n}, y_{1}, y_{2}, \ldots, y_{n}\right) \\
& u_{j}\left(y_{1}, y_{2}, \ldots, y_{n}\right) d y_{n} d y_{n-1} . . d y_{1}
\end{aligned}
$$

to get

$$
\begin{aligned}
& u_{j}\left(x_{1}, x_{2}, \ldots, x_{n}\right)=f_{i}\left(x_{1}, x_{2}, \ldots, x_{n}\right)+ \\
& N_{i}\left(x_{1}, x_{2}, \ldots, x_{n}\right)
\end{aligned}
$$

To solve equation(2) by Adomain's decomposition method, we let

$$
u_{j}\left(x_{1}, x_{2}, \ldots, x_{n}\right)=\sum_{m=0}^{\infty} u_{i, m}\left(x_{1}, x_{2}, \ldots, x_{n}\right)
$$

and

$$
N_{i}\left(x_{1}, x_{2}, \ldots, x_{n}\right)=\sum_{m=0}^{\infty} A_{i, m}
$$

where

$A_{i, m}, m=0,1, \ldots$ are polynomial depending on $u_{1,0}, u_{1,1} \ldots, u_{1, m}, \ldots, u_{n, 0}, u_{n, 1}, \ldots, u_{n, m}$ and they are called Adomian polynomials. Hence, equation (2) can be rewritten as:

$$
\begin{aligned}
& \sum_{\mathrm{m}=0}^{\infty} \mathrm{u}_{\mathrm{i}, \mathrm{m}}\left(\mathrm{x}_{1}, \mathrm{x}_{2}, \ldots, \mathrm{x}_{\mathrm{n}}\right)=\mathrm{f}_{\mathrm{i}}\left(\mathrm{x}_{1}, \mathrm{x}_{2}, \ldots, \mathrm{x}_{\mathrm{n}}\right)+ \\
& \sum_{\mathrm{m}=0}^{\infty} \mathrm{A}_{\mathrm{i}, \mathrm{m}}\left(\mathrm{u}_{1,0}, \mathrm{u}_{1,1} \ldots, \mathrm{u}_{1, \mathrm{~m}}, \ldots, \mathrm{u}_{\mathrm{n}, 0}, \mathrm{u}_{\mathrm{n}, 1}, \ldots, \mathrm{u}_{\mathrm{n}, \mathrm{m}}\right)
\end{aligned}
$$

From equation (3) we define :

$$
\begin{aligned}
& u_{i, 0}\left(x_{1}, x_{2}, \ldots, x_{n}\right)=f_{i}\left(x_{1}, x_{2}, \ldots, x_{n}\right), \\
& u_{i, m+1}\left(x_{1}, x_{2}, \ldots, x_{n}\right)= \\
& A_{i, m}\left(u_{1,0}, u_{1,1} \ldots, u_{1, m}, \ldots, u_{n, 0}, u_{n, 1}, \ldots, u_{n, m}\right), \\
& i=1,2, \ldots, n, m=0,1, \ldots
\end{aligned}
$$

To determine Adomain polynomials, we consider the expansions: 


$$
\begin{aligned}
& \mathrm{u}_{\mathrm{i}, \lambda}\left(\mathrm{x}_{1}, \mathrm{x}_{2}, \ldots, \mathrm{x}_{\mathrm{n}}\right)= \\
& \sum_{\mathrm{m}=0}^{\infty} \lambda^{\mathrm{m}} \mathrm{u}_{\mathrm{i}, \mathrm{m}}\left(\mathrm{x}_{1}, \mathrm{x}_{2}, \ldots, \mathrm{x}_{\mathrm{n}}\right), \\
& \mathrm{N}_{\mathrm{i}, \lambda}\left(\mathrm{x}_{1}, \mathrm{x}_{2}, \ldots, \mathrm{x}_{\mathrm{n}}\right)=\sum_{\mathrm{m}=0}^{\infty} \lambda^{\mathrm{m}} \mathrm{A}_{\mathrm{i}, \mathrm{m}}
\end{aligned}
$$

where $\lambda$ is a parameter introduced for convenience. From equation (6) we obtain:

$$
A_{i, m}=\frac{1}{m !}\left[\frac{d^{m}}{d \lambda^{m}} N_{i, \lambda}\left(u_{1}, u_{2}, \ldots, u_{n}\right)\right]_{\lambda=0} \ldots
$$

and from equations $(*),(5)$ and (7) we have:

$$
\begin{aligned}
& A_{i, m}\left(u_{1,0}, u_{1,1} \ldots, u_{1, m}, \ldots, u_{n, 0}, u_{n, 1}, \ldots, u_{n, m}\right)= \\
& \frac{1}{m !}\left[\frac{d^{m}}{d \lambda^{m}} \int_{a_{1}}^{b_{1} b_{2}} \int_{a n}^{b} \ldots \sum_{j=1}^{b n} k_{i, j}\left(x_{1}, x_{2}, \ldots, x_{n}, y_{1}, y_{2}, \ldots, y_{n}\right)\right. \\
& \left.u_{j}\left(y_{1}, y_{2}, \ldots, y_{n}\right) d y_{n} d y_{n-1} \ldots d y_{1}\right]_{\lambda=0} \\
& {\left[\int_{a_{1}}^{b_{1} 2} \int_{a n}^{b_{2}} \ldots \sum_{m=0}^{b n} k_{i, j}\left(x_{1}, x_{2}, \ldots, x_{n}, y_{1}, y_{2}, \ldots, y_{n}\right)\right.} \\
& \left.\sum_{m=0}^{n} \frac{1}{m !} \frac{d^{m}}{d \lambda^{m}} u_{i, m}\left(y_{1}, y_{2}, \ldots, y_{n}\right) d y_{n} d y_{n-1} \ldots d y_{1}\right]
\end{aligned}
$$

So, the solution of the system given by equation(1) will be as follows:

$$
\begin{aligned}
& u_{i, 0}\left(x_{1}, x_{2}, \ldots, x_{n}\right)=f_{i}\left(x_{1}, x_{2}, \ldots, x_{n}\right), \\
& u_{i, m+1}\left(x_{1}, x_{2}, \ldots, x_{n}\right)=\int_{a_{1}}^{b_{1}} \int_{a 2} \ldots \int_{a n}^{b n} \sum_{j=1}^{n} k_{i, j}\left(x_{1}, x_{2}, \ldots, x_{n},\right. \\
& \left.y_{1}, y_{2}, \ldots, y_{n}\right) u_{j, m}\left(y_{1}, y_{2}, \ldots, y_{n}\right) d y_{n} d y_{n-1} \ldots d y_{1}
\end{aligned}
$$

\section{3-Numerical Example}

In this section we give two examples of systems of multi-dimensional linear Fredholm integral equations of the second kind with their approximated solutions via Adomain decomposition method.

\section{Example (1):}

Consider the tow-dimensional linear Fredholm integral equation of the second kind:

$$
\mathrm{u}(\mathrm{x}, \mathrm{y})=\mathrm{xy}^{2}-\frac{1}{8} \mathrm{x}+\int_{0}^{1} \int_{0}^{1} \mathrm{xmu}(\mathrm{z}, \mathrm{m}) \mathrm{dzdm}
$$

This example is constructed such that the exact solution of it is $u(x, y)=x y^{2}$. Here we use Adomain decomposition method to find the solutions $u$. To do this we use the following Adomain scheme:

$$
u_{0}(x, y)=x y^{2}-\frac{1}{8} x
$$

and

$$
\mathrm{u}_{\mathrm{r}+1}(\mathrm{x}, \mathrm{y})=\int_{0}^{1} \int_{0}^{1} \mathrm{xmu} \mathrm{r}_{\mathrm{r}}(\mathrm{z}, \mathrm{m}) \mathrm{dzdm}, \mathrm{r}=1,2, \ldots
$$

For the first iteration, we have:

$$
\begin{aligned}
\mathrm{u}_{1}(\mathrm{x}, \mathrm{y}) & =\int_{0}^{1} \int_{0}^{1} \mathrm{xmu}_{0}(\mathrm{z}, \mathrm{m}) \mathrm{dzdm} \\
& =\frac{3}{32} \mathrm{x} .
\end{aligned}
$$

Therefore the approximated solution of this example with two terms is:

$$
\begin{aligned}
\mathrm{Q}_{2}(\mathrm{x}, \mathrm{y}) & =\mathrm{u}_{0}(\mathrm{z}, \mathrm{m})+\mathrm{u}_{1}(\mathrm{z}, \mathrm{m}) \\
& =\mathrm{xy}^{2}-\frac{1}{8} \mathrm{x}+\frac{3}{32} \mathrm{x} \\
& =\mathrm{xy}^{2}-\frac{1}{32} \mathrm{x} .
\end{aligned}
$$

For the second iteration, we have:

$$
\begin{aligned}
\mathrm{u}_{2}(\mathrm{x}, \mathrm{y}) & =\int_{0}^{1} \int_{0}^{1} \mathrm{xmu}_{1}(\mathrm{z}, \mathrm{m}) \mathrm{dzdm} \\
& =\frac{1}{32} \mathrm{x} .
\end{aligned}
$$

Therefore the approximated solution of this example with three terms is:

$$
\begin{aligned}
Q_{3}(x, y) & =u_{0}(x, y)+u_{1}(x, y)+u_{2}(x, y) \\
& =x y^{2}-\frac{1}{8} x+\frac{3}{32} x+\frac{1}{32} x \\
& =x y^{2}
\end{aligned}
$$

Not that $Q_{3}(x, y)=x y^{2}$ is the exact solution of this example.

\section{Example (2):}

Consider the system of the twodimensional linear Fredholm integral equations:

$$
\begin{aligned}
& u_{1}(x, y)=\frac{7}{12} x y+\int_{0}^{1} \int_{0}^{1} x y\left(u_{1}(z, m)+u_{2}(z, m)\right) d z d m \\
& u_{2}(x, y)=\frac{17}{24} x^{2} y+\int_{0}^{1} \int_{0}^{1} x^{2} y z\left(u_{1}(z, m)+u_{2}(z, m)\right) d z d m
\end{aligned}
$$

This example is constructed such that the exact solution of it is

$$
u_{1}(x, y)=x y \text { and } u_{2}(x, y)=x^{2} y .
$$


Here we use Adomain decomposition method to find the solutions $u_{1}, u_{2}$ of this example. To do this we use the following Adomain scheme:

$$
\begin{aligned}
& \begin{aligned}
u_{1,0}(x, y) & =\frac{7}{12} x y \\
& \approx 0.5833 x y
\end{aligned} \\
& \begin{aligned}
u_{2,0}(x, y) & =\frac{17}{24} x^{2} y \\
& \approx 0.7083 x^{2} y
\end{aligned} \\
& \text { and } \begin{aligned}
\mathrm{u}_{1, \mathrm{r}+1}(\mathrm{x}, \mathrm{y})=\int_{0}^{1} \int_{0}^{1} \mathrm{xy}\left(\mathrm{u}_{1, \mathrm{r}}(\mathrm{z}, \mathrm{m})+\mathrm{u}_{2, \mathrm{r}}(\mathrm{z}, \mathrm{m})\right) \mathrm{dzdm}, \mathrm{r}=1,2, \ldots \\
\mathrm{u}_{2, \mathrm{r}+1}(\mathrm{x}, \mathrm{y})=\int_{0}^{1} \int_{0}^{1} \mathrm{x}^{2} \mathrm{yz}\left(\mathrm{u}_{1, \mathrm{r}}(\mathrm{z}, \mathrm{m})+\mathrm{u}_{2, \mathrm{r}}(\mathrm{z}, \mathrm{m})\right) \mathrm{dzdm}, \mathrm{r}=1,2, \ldots
\end{aligned}
\end{aligned}
$$

For the first iteration, we have:

$$
\begin{aligned}
\mathrm{u}_{1,1}(\mathrm{x}, \mathrm{y}) & =\int_{0}^{1} \int_{0}^{1} \mathrm{xy}\left(\mathrm{u}_{1,0}(\mathrm{z}, \mathrm{m})+\mathrm{u}_{2,0}(\mathrm{z}, \mathrm{m})\right) \mathrm{dzdm} \\
& =\frac{9}{72} \mathrm{xy} \\
& \approx 0.2639 \mathrm{xy} . \\
\mathrm{u}_{2,1}(\mathrm{x}, \mathrm{y}) & =\int_{0}^{1} \int_{0}^{1} \mathrm{x}^{2} \mathrm{yz}\left(\mathrm{u}_{1,0}(\mathrm{z}, \mathrm{m})+\mathrm{u}_{2,0}(\mathrm{z}, \mathrm{m})\right) \mathrm{dzdm} \\
& =\frac{107}{576} \mathrm{x}^{2} \mathrm{y} \\
& \approx 0.1858 \mathrm{x}^{2} \mathrm{y} .
\end{aligned}
$$

Therefore the approximated solutions of this example with two terms are:

$$
\begin{aligned}
\mathrm{Q}_{1,2}(\mathrm{x}, \mathrm{y}) & =\mathrm{u}_{1,0}(\mathrm{x}, \mathrm{y})+\mathrm{u}_{1,1}(\mathrm{x}, \mathrm{y}) \\
& =\frac{7}{12} \mathrm{xy}+\frac{19}{72} \mathrm{xy} \\
& \approx 0.5833 \mathrm{xy}+0.2639 \mathrm{xy} \\
& \approx 0.8472 \mathrm{xy} \\
\mathrm{Q}_{2,2}(\mathrm{x}, \mathrm{y}) & =\mathrm{u}_{2,0}(\mathrm{x}, \mathrm{y})+\mathrm{u}_{2,1}(\mathrm{x}, \mathrm{y}) \\
& =\frac{17}{24} \mathrm{x}^{2} \mathrm{y}+\frac{107}{576} \mathrm{x}^{2} \mathrm{y} \\
& \approx 0.7083 \mathrm{x}^{2} \mathrm{y}+0.1858 \mathrm{x}^{2} \mathrm{y} \\
& \approx 0.8941 \mathrm{x}^{2} \mathrm{y}
\end{aligned}
$$

For the second iteration, we have:

$$
\begin{aligned}
\mathrm{u}_{1,2}(\mathrm{x}, \mathrm{y}) & =\int_{0}^{1} \int_{0}^{1} \mathrm{xy}\left(\mathrm{u}_{1,1}(\mathrm{z}, \mathrm{m})+\mathrm{u}_{2,1}(\mathrm{z}, \mathrm{m})\right) \mathrm{dzdm} \\
& =\frac{335}{3456} \mathrm{xy} \\
& \approx 0.0969 \mathrm{xy} . \\
\mathrm{u}_{2,2}(\mathrm{x}, \mathrm{y}) & =\int_{0}^{1} \int_{0}^{1} \mathrm{x}^{2} \mathrm{yz}\left(\mathrm{u}_{1,1}(\mathrm{z}, \mathrm{m})+\mathrm{u}_{2,1}(\mathrm{z}, \mathrm{m})\right) \mathrm{dzdm} \\
& =\frac{929}{13824} \mathrm{x}^{2} \mathrm{y} \\
& \approx 0.0672 \mathrm{x}^{2} \mathrm{y} .
\end{aligned}
$$

Therefore the approximated Solutions of this example with three terms are:

$$
\begin{aligned}
Q_{1,3}(x, y)= & u_{1,0}(x, y)+u_{1,1}(x, y)+u_{1,2}(x, y) \\
& =\frac{7}{12} x y+\frac{19}{72} x y+\frac{335}{3456} x y \\
& =\frac{3263}{3456} x y \\
& \approx 0.9442 x y . \\
Q_{2,3}(x, y)= & u_{2,0}(x, y)+u_{2,1}(x, y)+u_{2,2}(x, m) \\
& =\frac{17}{24} x^{2} y+\frac{107}{576} x^{2} y+\frac{929}{13824} x^{2} y \\
& =\frac{13289}{13824} x^{2} \\
& \approx 0.9613 x^{2} y .
\end{aligned}
$$

In the same way, the components $\mathrm{Q}_{1, \mathrm{k}}(\mathrm{x}, \mathrm{y})$ and $\mathrm{Q}_{2, \mathrm{k}}(\mathrm{x}, \mathrm{y})$ can be calculated for $\mathrm{k}=4$, $5, \ldots$ The solutions with ten terms are given as:

$$
\begin{aligned}
\mathrm{Q}_{1,10}(\mathrm{x}, \mathrm{y}) & =\sum_{\mathrm{i}=0}^{9} \mathrm{u}_{1, \mathrm{i}}(\mathrm{x}, \mathrm{y}) \\
& =\frac{47544222539155}{47552535724032} \mathrm{xy} \\
& \approx 0.9998 \mathrm{xy} . \\
\mathrm{Q}_{2,10}(\mathrm{x}, \mathrm{y}) & =\sum_{\mathrm{i}=0}^{9} \mathrm{u}_{2, \mathrm{i}}(\mathrm{x}, \mathrm{y}) \\
& =\frac{63395395174439}{63403380965376} \mathrm{x}^{2} \mathrm{y} \\
& \approx 0.9999 \mathrm{x}^{2} \mathrm{y} .
\end{aligned}
$$

\section{4- Conclusion}

As seen before Admian decomposition method have been successfully employedto obtain the approximated solutions of systems of the multi-dimensional linear Fredholm integral equations of the second kind. More 
accurate results can be obtained by increasing the number of iteration. On the other hand, finding the approximated solutions of systems of the multi-dimensional nonlinear Fredholm integral equations of the second kind by using Adomian decomposition method is a good subject for further research.

\section{5-References}

[1] k. Abbaoui and Y. Cherruault, "Convergence of Adomian's Applied to Differential Equations",Math1, Comput, Model, Vol. 28, No.5, 1994, pp. 103-110.

[2] G. Adomian, "Solving Frontier Problems of Physics", Decomposition Method Kluwer, 1994.

[3] E. Babolian, J. Biazar and A. Vahidi, "On the Decomposition Method for System of Linear Equations and System of Linear Volterra Integral Equation", Appl. Math. Comput.,Vol. 147, 2004, pp.19-27.

[4] J. Biazar, E. Babolian and R. Islam, "Solution of A System of Volterra Integral Equations of the First Kind by Adomian Method", Appl. Math. Comput., Vol. 139, 2003, pp.249-258.

[5] Y. Cherruault and G. Saccomandi, "New Results for Convergence of Adomian Method Applied to Integral Equations", Math1. Comput. Model. Vol.16, No. 2, 1992, pp. 83-93.

[6] M. Shaher and Kamel Al-Khaled, "Numerical Solutions for Systems of Fractional Differential Equations by the DecompositionMethod",Appl. Math. Comput., Vol. 162, 2005, pp. 1351-1365.

[7] A. Vahidi and M. Mokhtari, "On the Decomposition Method for System of Linear Equations Fredholm Integral Equations of the Second Kind", Appl. Math. Scien., Vol.2, No.2, 2008, pp.5762. 